\title{
Efectividad de los extractos vegetales de orégano silvestre (Lippia origanoides K.) y citronela (Cymbopogon citratus D.C.) sobre Rhyzopertha dominica (F.) (Coleoptera: Bostrichidae)
}

\section{Effectiveness of plant extracts of wild oregano (Lippia origanoides $\mathbf{K}$.) and citronela (Cymbopogon citratus D.C.) on Rhyzopertha dominica (F.) (Coleoptera: Bostrichidae)}

\author{
Luisiris Flores ${ }^{1}$; Yohan Solano ${ }^{2 *}$; María Elena Sanabria ${ }^{3}$; Dilcia Hernández ${ }^{4}$
}

\section{RESUMEN}

Los extractos vegetales foliares han sido usados como plaguicidas y/o repelentes contra bacterias, hongos y artrópodos. La actividad biológica de estos preparados se debe a los metabolitos secundarios (MS), compuestos que las plantas sintetizan y cuya concentración varía aún en sus órganos. Se determinó por cromatografía de capa fina la concentración de los grupos de MS en extractos etanólicos (EE) obtenidos a partir de hojas de orégano (Lippia origanoides K.) y citronela (Cymbopogon citratus D.C.) y se evaluó el efecto de los mismos sobre Rhyzopertha dominica (F.) a las concentraciones de 40, 60, 80, 85 y $95 \%$ para ambos $\mathrm{EE}$, en un diseño completamente al azar con arreglo factorial $2 \times 5$. El estudio fitoquímico permitió determinar la presencia en los EE de orégano y citronela, de aceites esenciales, saponinas $(2,02 \mathrm{~mm}$ y $3,46 \mathrm{~mm})$, alcaloides $(0,0975$ y $0,1223 \mu \mathrm{L} / \mathrm{mL})$, fenoles $(0,0899$ y $0,11375 \mu \mathrm{L} / \mathrm{mL})$ y flavonoides $(0,1055$ y $0,1312 \mu \mathrm{L} / \mathrm{mL})$, respectivamente. Se presentaron además diferencias significativas $(P<0,01)$ entre las concentraciones evaluadas, obteniéndose al 95\% valores de mortalidad de 56,75\%. De igual modo, se presentaron diferencias significativas entre la mortalidad ocasionada por ambos $\mathrm{EE}(P<0,05)$, donde el porcentaje de mortalidad a la concentración de $60 \%$, fue mayor con el EE de orégano (35,5\%) que con el de citronela (16\%), mientras que a dosis mayores, la efectividad de éste último fue siempre superior a la de orégano. Los MS en los EE resultaron efectivos ocasionando valores aceptables de mortalidad en $R$. dominica, a altas concentraciones, recomendándose su uso como control preventivo.

Palabras clave: insecticida, metabolitos secundarios, mortalidad.

\begin{abstract}
Vegetal extracts from leaves have been used as pesticides and/or repellents against bacteria, fungi and arthropods. The biological activity of this extracts is due to presence of secondary metabolites (SM), which are synthesized by plants and its concentration varies even in their organs. The concentration of SM groups was determined by layer chromatography in ethanolic extracts (EE) obtained from leaves of oregano (Lippia origanoides K.) and citronella (Cymbopogon citratus D.C.) and the effect of them was evaluated on Rhyzopertha dominica $(F$.) at concentration of 40,60,80, 85 and $95 \%$ for both of EE, in a random design with factorial array $2 \times 5$. The phytochemistry study allowed determine presence in EE of oregano and citronella of essential oil, saponins (2.02 $\mathrm{mm}$ and $3.46 \mathrm{~mm})$, alkaloids $(0.0975$ and $0.1223 \mu \mathrm{L} / \mathrm{mL})$, phenols $(0.0899$ and $0.11375 \mu \mathrm{L} / \mathrm{mL})$ and flavonoids $(0.1055$ and $0.1312 \mu \mathrm{L} / \mathrm{mL})$, respectively. There were significant differences $(\mathrm{P}<0.01)$ between concentrations evaluated, where dose of $95 \%$ caused $56.75 \%$ of mortality. In the same way, significant differences between mortality caused for both of $E E(\mathrm{P}<0.05)$ were registered, where the mortality percentage at concentration of $60 \%$, was higher with EE of oregano (35.5\%) than with of citronella (16\%), while the effectiveness of the latter was always superior to that of oregano, higher doses. SM's in EE were effective producing acceptable values of mortality in $\mathrm{R}$. dominica, to high doses, and its use is recommended as preventive control.
\end{abstract}

Key words: insecticide, secondary metabolites, mortality.

1 Programa de Ingeniería Agroindustrial. Decanato de Agronomía. Universidad Centroccidental Lisandro Alvarado. Lara, Venezuela.

2 Departamento de Ecología y Control de Calidad. Decanato de Agronomía. Universidad Centroccidental Lisandro Alvarado. Lara, Venezuela.

3 Posgrado de Agronomía. Decanato de Agronomía. Universidad Centroccidental Lisandro Alvarado. Lara, Venezuela.

4 Departamento de Ciencias Biológicas. Decanato de Agronomía. Universidad Centroccidental Lisandro Alvarado. Lara, Venezuela.

* Autor para correspondencia: ysolano@ucla.edu.ve

Fecha de Recepción: 27 Junio, 2017.

Fecha de Aceptación: 23 Octubre, 2017.

DOI: 


\section{Introducción}

El ataque de insectos a granos y alimentos como harinas, galletas y pastas, es una de las principales causas de pérdida de estos productos cuando se encuentran en almacenamiento. El daño producido por estas plagas está asociado a la reducción del peso, deterioro del poder y energía germinativa de las semillas, disminución del valor comercial, contaminación por los residuos corporales y de su metabolismo, y la aparición de hongos (Alonso et al., 2009).

En el control de los insectos plaga en la agroindustria, los insecticidas ha sido los más utilizados debido a su rápida acción en reducir las poblaciones; sin embargo, los productos químicos dejan residuos tóxicos que afectan la salud de consumidores, así como también promueven la aparición de resistencia genética, cuando su uso es indiscriminado (Devine et al., 2005). Es por ello que diversas investigaciones han evaluado alternativas ecológicas de control como el uso de metabolitos secundarios (MS) contenidos en extractos vegetales (EV) (Olivero et al., 2009; Lima et al., 2013; Pino et al., 2013; Priya et al., 2016).

Los metabolitos secundarios son compuestos sintetizados por las especies vegetales durante su desarrollo, sin embargo las plantas expuestas a condiciones adversas como el ataque de herbívoros y de microorganismos como virus, bacterias, hongos; competencia por espacio, luz y nutrientes u otro tipo de estrés biótico o abiótico (salinidad, temperatura, entre otros), tienden a aumentar la concentración de los MS (Sepúlveda et al., 2004; Celis et al., 2008).

Entre los principales grupos de MS se encuentran los terpenos, compuestos fenólicos, glicósidos y alcaloides (Avalos y Pérez 2009), los cuales pueden estar contenidos en aceites esenciales (AE), extractos acuosos (EA), extractos etanólicos (EE) o polvos vegetales (PV). Estos extractos pueden tener efecto insectistático o repelente ya que son capaces de inhibir el desarrollo de los insectos al afectar su alimentación (atracción o fagodepresión), el sistema nervioso (desorientación), el comportamiento reproductivo (inhibición de la oviposición) y/o produciendo mortalidad cuando resultan altamente tóxicos (Sepúlveda et al., 2003; Celis et al., 2008; Pino et al., 2013).
El orégano silvestre (Lippia origanoides K.) y la citronela (Cymbopogon citratus D.C) son especies vegetales utilizadas como potenciadores de sabor y con fines medicinales, que han sido estudiadas por el efecto que producen en insectos plaga como T. castaneum y Sitophilus zeamais (Mots.) (Olivero et al., 2009; Lima et al., 2013). Entre los metabolitos registrados para $L$. origanoides se encuentran los terpenos, alcaloides, cetona monoterpenica, flavonoides y fenilpropanoides (Castañeda et al., 2007), mientras que para $C$. citratus se han señalado los terpenos, alcaloides y compuestos fenólicos (Avoseh et al., 2015).

La especie Rhyzopertha dominica F., conocida como barrenador de los granos, se encuentra distribuida alrededor del mundo y es considerada la principal plaga del trigo, el maíz y el arroz almacenado (Alonso et al., 2009). Este insecto ha desarrollado resistencia a insecticidas (Schlipalius et al., 2002; Rajendran y Gunasekaran, 2002), por lo cual diversas investigaciones han evaluado los EV como alternativas para su control (Reyes et al., 2012; Khaliq et al., 2014; Priya et al., 2016). En este sentido, la presente investigación se condujo con la finalidad de evaluar la efectividad de los EV foliares de orégano silvestre y citronela sobre $R$. dominica, una de las especies más perjudiciales en granos y productos almacenados.

\section{Materiales y Métodos}

\section{Colecta y cría de $R$. dominica}

Los adultos del barrenador fueron obtenidos de granos de maíz infestado proveniente de crías del Laboratorio de Biología y Fisiología Poscosecha del programa de Ingeniería Agroindustrial de la Universidad Centroccidental Lisandro Alvarado, Lara, Venezuela. Se seleccionaron grupos de 50 adultos de $R$. dominica no sexados y de edades mezcladas, los cuales se colocaron sobre 250 gramos de maíz amarillo en envases de $500 \mathrm{ml}$ de capacidad, tapados con un trozo de tela de organdí sujetada con una banda de goma para permitir la ventilación. Este procedimiento se repitió hasta completar $3 \mathrm{~kg}$ de maíz infestado, con la finalidad de garantizar un suministro permanente de adultos. 
Los insectos se mantuvieron en los frascos por 3 semanas para permitir su reproducción y oviposición. Posteriormente, los adultos parentales fueron removidos de los envases para esperar la emergencia de los individuos que conformaron la primera generación (F1), los cuales fueron aislados con 2 o 3 semanas de edad y usados en las pruebas biológicas.

\section{Obtención de los extractos etanólicos de Lippia origanoides y Cymbopogon citratus}

Se colectaron hojas frescas y aparentemente sanas de orégano silvestre y citronela, de plantas ubicadas en el campo experimental del Posgrado de Agronomía de la UCLA; las mismas fueron secadas bajo sombra, se molieron en una licuadora convencional Oster ${ }^{\circledR}$ y se maceraron en frascos de vidrio con etanol (96\%) durante 24 horas. Posteriormente, el solvente fue separado por destilación, utilizando un rotavapor Brinkmann y el extracto crudo fue envasado en frascos estériles ámbar y almacenados bajo refrigeración a $8 \pm 2{ }^{\circ} \mathrm{C}$, hasta su utilización en los análisis fitoquímicos y aplicación en los experimentos.

Determinación de metabolitos secundarios en los extractos etanólicos de Lippia origanoides y Cymbopogon citratus

\section{Determinación cualitativa}

La determinación de alcaloides, fenoles y flavonoides en los EE foliares de orégano silvestre y citronela, se realizó por cromatografía de capa fina utilizando la metodología de Marcano y Hasegawa (2002). Para ello, se usaron dos cromatofolios de sílica gel (Merk® 6,5 cm de largo x 2,5 cm de ancho) y en cada uno de ellos se colocaron dos gotas $(20 \mu \mathrm{L})$ del EE correspondiente a $1 \mathrm{~cm} \mathrm{de}$ uno de sus extremos, utilizando una micropipeta. Seguidamente, los cromatofolios se colocaron en cámaras cromatográficas que contenían el eluyente específico para cada grupo de MS a separar: n-butanol + ácido acético + agua (9:2:1), agua + ácido acético (9:1) y benceno + ácido acético + agua (12:7:2). Para el revelado de las cromatografías se consideró el color naranja intenso como indicativo de alcaloides, mientras que la presencia de fenoles fue mostrada por una coloración parda oscura cuando se rocía cloruro férrico al 1\%, y para flavonoides el color blanco fluorescente, respectivamente.

Los aceites esenciales se determinaron por el olor característico que confieren estos MS a cada EE evaluado, mientras que las saponinas se determinaron mezclando $1 \mathrm{ml}$ de cada extracto con $1 \mathrm{ml}$ de agua destilada, seguidamente se tomó $1 \mathrm{ml}$ de la mezcla y se colocó en un tubo de ensayo para agitar vigorosamente hasta la formación de espuma. La persistencia de ésta, luego de $15 \mathrm{~min}$, fue considerada como positiva para saponinas y su contenido fue valorado de acuerdo a los rangos señalados por Cuellar et al., (1999): $0 \mathrm{~mm}$ (negativo); 0,1 - $5 \mathrm{~mm}$ (muy bajo); 5,1 - 9 (bajo); 9,1 - 14 (moderado) y mayor de $14 \mathrm{~mm}$ (alto).

\section{Determinación cuantitativa}

Las corridas de cromatografía hechas para la determinación cualitativa fueron usadas para la cuantificación de los grupos de MS utilizando la metodología descrita por Vásquez et al. (2008). Para ello, se marcó el área ocupada por el MS en el cromatofolio de silica gel y con ayuda de un perforador se extrajeron tres secciones: el área conocida (AC) (MS + sílica + solvente), el área desconocida (AD) (espacio restante del área recorrida por el metabolito: MS + sílica + solvente, la cual se raspo y peso), y el área testigo (ACt) (cromatofolio testigos con sílica + solvente los cuales se perforaron y rasparon). Cada una de las muestras se pesó en una balanza analítica Ohaus Adventure ${ }^{\mathrm{TM}} \mathrm{N}^{\mathrm{o}}$ AR2140, y los datos fueron introducidos en la siguiente ecuación:

$$
\mu \mathrm{g} \text { de } \frac{\mathrm{MS}}{\mathrm{ml}}=\frac{\left[\left(\left(\left(\mathrm{AD} \times \frac{\mathrm{AC}-\mathrm{ACt}}{\mathrm{AC}}\right)+(\mathrm{AC}+\mathrm{ACt})\right) * 1000\right) * 1000\right]}{20 \mu 1}
$$


Donde MS representa a alcaloides, fenoles o flavonoides, respectivamente, y $20 \mu \mathrm{l}$ el volumen del extracto que se agregó a cada cromatofolio.

Efecto de los extractos etanólicos de Lippia origanoides $y$ Cymbopogon citratus sobre Rhyzopertha dominica

Se seleccionaron granos de maíz enteros, aparentemente sanos, sin grietas y limpios, se pesaron en una balanza electrónica KERNMR, Modelo EMB220-1, para conformar muestras de 20 g. Seguidamente, cada una se colocó en una bandeja plástica y se asperjaron con $1 \mathrm{ml}$ del tratamiento correspondiente (EE de orégano o citronela a concentraciones de 40,60,80, 85 y $95 \%$ y un testigo, para el cual solo se agregó agua, y se esperó por un periodo de 5 min para permitir la impregnación de los granos con el extracto.

Una vez impregnados, se colocaron en envases de plástico de $120 \mathrm{~g}$ de capacidad y se introdujeron 20 adultos de $R$. dominica, no sexados y con edades comprendidas entre 2 a 3 semanas. Cada envase se selló con una tapa que tenía un orificio $(2 \mathrm{~cm}$ de diámetro) cubierto con un trozo de tela organdí para permitir la ventilación, y se observaron cada 24 horas hasta completar 96 horas. En cada observación, los envases fueron destapados para contar la cantidad de insectos vivos (con movilidad) o muertos (detención completa de movimiento y/o comportamiento y movimiento descoordinados). Los adultos de $R$. dominica vivos, fueron retornados al envase con granos de maíz tratado, mientras que los considerados como muertos se aislaron en nuevos envases con granos de maíz no tratado y se mantuvieron en observación por 24 horas, para comprobar la muerte de los mismos. Cuando éstos permanecían vivos, fueron regresados al envase con el tratamiento correspondiente.

Cada tratamiento fue replicado 5 veces y cada repetición estuvo conformada por dos unidades experimentales. Se siguió un diseño completamente al azar con arreglo factorial $2 \times 5$, donde el primer factor correspondió a los EE y el segundo a las cinco concentraciones evaluadas. El efecto insecticida de los extractos etanólicos sobre adultos de $R$. dominica, se consideró como una relación directa con el porcentaje de insectos muertos.

El porcentaje de mortalidad fue corregido de acuerdo a la fórmula de Abbott (1925), la cual describe:

$$
\mathrm{Pc}=\left(\frac{\mathrm{P} \exp -\mathrm{P} \text { cont }}{100 \%-\text { cont }}\right) \times 100
$$

Donde Pc representó el porcentaje de mortalidad corregido, Pexp el porcentaje de mortalidad experimental, y Pcont el porcentaje de mortalidad control.

\section{Análisis de datos}

Los datos de porcentaje de mortalidad fueron sometidos a análisis de varianza y posteriormente a pruebas de medias según Tukey utilizando el paquete estadístico Statistix versión 10.0. Los porcentajes de mortalidad fueron transformados a arcoseno ya que estos presentaron una distribución binomial.

\section{Resultados y Discusión}

\section{Determinación y cuantificación de los grupos de metabolitos secundarios en los extractos etanólicos de orégano silvestre (Lippia origanoides) y citronela (Cymbopogon citratus)}

Se evidenció la presencia de alcaloides, fenoles, flavonoides, aceites esenciales y saponinas en los EE de orégano y citronela, y se determinó que estos grupos de MS se encontraban menos concentrados en el primero que en el segundo (Tabla 1). Estos resultados coincidieron con los señalados por Alvarado et al. (2011), Sivira et al. (2011) y Fernández et al. (2016) quienes detectaron la presencia de estos MS en las mismas especies vegetales. Sin embargo, contrario a estos resultados, Bolívar et al. (2009) no reportaron la presencia de saponinas en el EE de orégano silvestre.

La presencia de estos grupos de MS en los extractos evaluados, permite asociarlos con los mecanismos de defensa contra insectos, ya que los alcaloides tienen la capacidad de bloquear neuroreceptores intermediarios y canales iónicos (Sepúlveda et al., 2003) y los fenoles intervienen en el desarrollo y obstrucción de las vías respiratorias del insecto (Sepúlveda et al., 2003; Celis et al., 2008). Por su parte, los flavonoides inhiben el transporte de electrones en las mitocondrias generando una reducción del consumo de oxígeno que provoca convulsiones y muerte (Celis et al., 2008), mientras que las saponinas tienen efecto disuasorio de alimentación, retraso en el desarrollo 
Tabla 1. Metabolitos secundarios encontrados en los extractos etanólicos de Lippia origanoides y Cymbopogon citratus

\begin{tabular}{|c|c|c|c|c|}
\hline \multirow{2}{*}{$\begin{array}{l}\text { Metabolitos } \\
\text { Secundarios }\end{array}$} & \multicolumn{2}{|c|}{ Lippia origanoides } & \multicolumn{2}{|c|}{ Cymbopogon citratus } \\
\hline & $\begin{array}{c}\text { Presencia (+) } \\
\text { Ausencia (-) }\end{array}$ & $\begin{array}{c}\text { Concentración } \\
(\mu \mathrm{L} / \mathrm{mL})\end{array}$ & $\begin{array}{c}\text { Presencia (+) } \\
\text { Ausencia (-) }\end{array}$ & $\begin{array}{c}\text { Concentración } \\
(\mu \mathrm{L} / \mathrm{mL})\end{array}$ \\
\hline Alcaloides & + & 0,0975 & + & 0,1223 \\
\hline Fenoles & + & 0,0899 & + & 0,11375 \\
\hline Flavonoides & + & 0,1055 & + & 0,1312 \\
\hline Saponinas & + & $2,02 \mathrm{~mm}$ & + & $3,46 \mathrm{~mm}$ \\
\hline Aceites esenciales & + & - & + & - \\
\hline
\end{tabular}

y disminución de la reproducción en los insectos (De Geyter et al., 2007).

Diversas investigaciones han indicado que los MS presentes en L. origanoides y C. citratus pueden actuar como barreras protectoras contra insectos al obstruir sus vías respiratorias por olores tóxicos en sus aceites esenciales, así como también pueden tener efecto disuasorio de la alimentación y/o causar la muerte (Antolinez et al., 2008; Acevedo et al., 2013).

\section{Efecto de los extractos etanólicos de Lippia origanoides y Cymbopogon citratus sobre la mortalidad de adultos de $R$. dominica}

Las concentraciones de los EE de orégano y citronela $(40,60,80,85$ y $95 \%)$ produjeron un efecto significativo sobre la mortalidad en adultos de $R$. dominica mostrando diferencias entre ellas $(\mathrm{P}<0,01)$ (Tabla 2). La Figura 1 muestra que la mortalidad del barrenador incrementó a medida que aumentó la dosis, no obstante el porcentaje de mortalidad producido al $95 \%$, ligeramente superó el $50 \%$ de la población estudiada. El efecto de los EE al 80 y $85 \%$ no fue significativamente diferente $(\mathrm{P}<0,01)$ con valores de mortalidad de
45 y $44 \%$, respectivamente. Los porcentajes más bajos de mortalidad se ubicaron en 25,75 y $10,5 \%$ a las dosis de 60 y 40\%, respectivamente.

En el ANOVA (Tabla 2) también se indican diferencias significativas $(\mathrm{P}<0,05)$ en la interacción entre los factores concentración por extracto, para la variable porcentaje de mortalidad de $R$. dominica, la cual se afectó en forma directa con el incremento de las concentraciones de los EE de orégano y citronela (Figura 2). El mayor porcentaje de mortalidad del barrenador (65\%) fue producido por el EE de citronela al 95\%, el cual fue significativamente diferente del producido por el orégano $(48,5 \%)$ a la misma dosis. El efecto de ambos EE al 80 y $85 \%$ fue también significativamente diferente $(\mathrm{P}<0,01)$ entre ambos extractos, y siempre mayor en citronela que en orégano.

A la dosis de $60 \%$, la mortalidad presentó diferencias estadísticas, siendo mayor para el orégano $(35,5 \%)$ que para citronela $(16 \%)$. Las concentraciones de 60,80 y $85 \%$ del EE de orégano no registraron diferencias significativas en el porcentaje de mortalidad, mientras que la dosis de $40 \%$ de ambos extractos mostró la mortalidad más baja de las dosis evaluadas.

Resultados similares fueron obtenidos por Gandhi y Pillai (2011) cuando aplicaron hojas

Tabla 2. ANOVA del efecto de las concentraciones de los extractos etanólicos de Lippia origanoides y Cymbopogon citratus sobre el porcentaje de mortalidad de adultos de $R$. dominica

\begin{tabular}{lccccc}
\hline \multicolumn{1}{c}{ Fuente } & Grados de libertad & Suma de cuadrados & Cuadrados medios & $\mathrm{F}$ & $\mathrm{P}$ \\
\hline Concentración & 4 & 0,26092 & 0,06523 & 16,52 & $0,0000^{* *}$ \\
Extracto & 1 & 0,00139 & 0,00139 & 0,35 & $0,5563 \mathrm{~ns}$ \\
Concentración* extracto & 4 & 0,04646 & 0,01162 & 2,94 & $0,0320^{*}$ \\
Error & 40 & 0,15798 & 0,00395 & & \\
Total & 49 & 0,46675 & & & \\
\hline
\end{tabular}

* Significativo a $\mathrm{P}<0,05$; ** Significativo a $\mathrm{P}<0,01$. ns: no significativo.

C.V. 24.45 


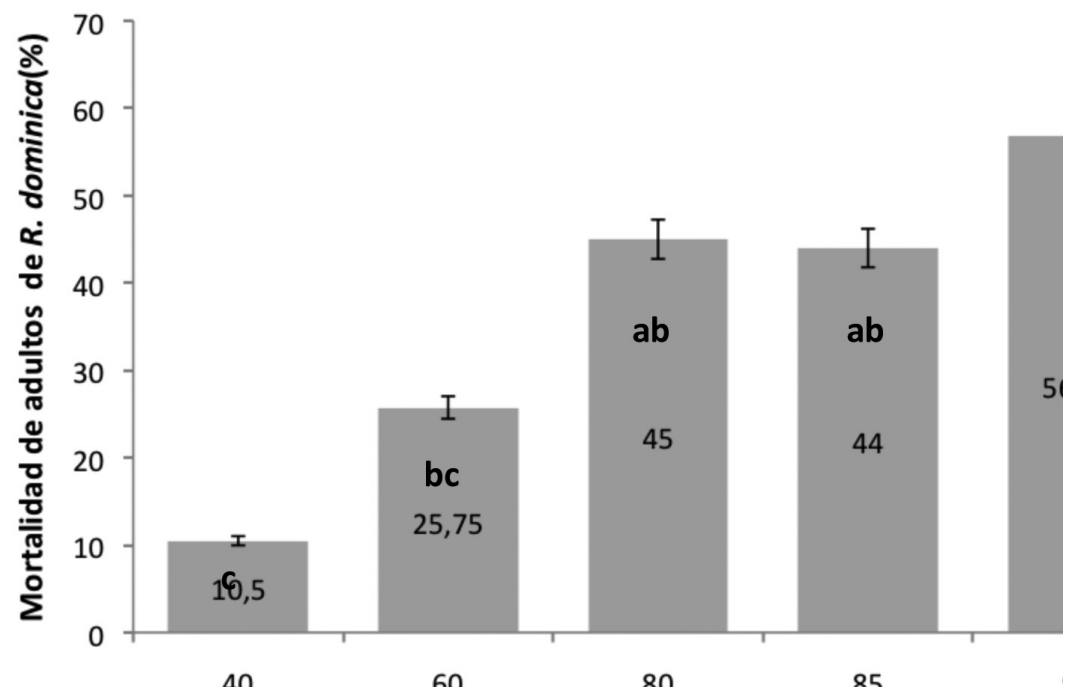

Figura 1. Mortalidad (\%) promedio de adultos de $R$. dominica ante el efecto de las concentraciones de los extractos etanólicos de Lippia origanoides y Cymbopogon citratus (Promedios con la misma letra no difieren estadísticamente, Tukey $\mathrm{P}<0,01$ )

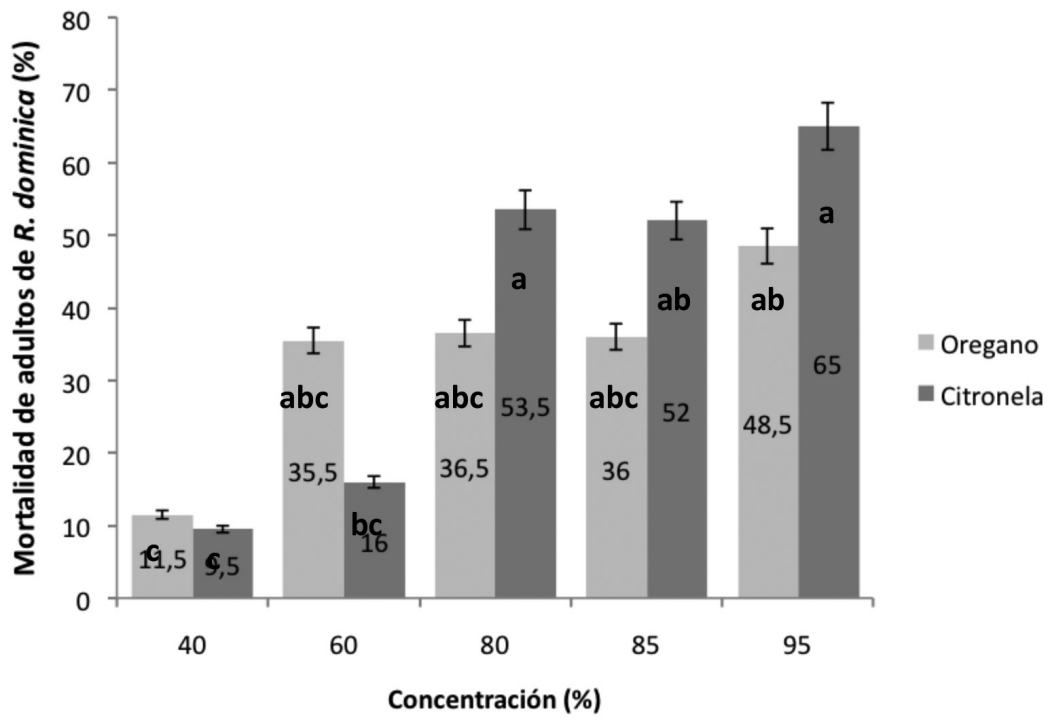

Figura 2. Mortalidad (\%) promedio de adultos de $R$. dominica ante el efecto de los extractos etanólicos de orégano y citronela, evaluados a cinco concentraciones. (Promedios con la misma letra no difieren estadísticamente, Tukey $\mathrm{P}<0,01$ )

pulverizadas de granada (Punica granatum (L.)) y curry (Murraya koenigii L.) sobre granos de trigo (79\% - 65\%) y arroz (79\%) ofrecido a $R$. dominica, respectivamente, argumentando que las diferencias se debieron a la protección que brindó el grano de trigo al insecto. De igual forma, Khaliq et al. (2014) señalaron que la mortalidad del barrenador incrementó de 40,19 hasta 50,36\% en la medida que la dosis del extracto de acetona de berberia (Nerium oleander L.) aumentó de 5 a $15 \%$, mientras que Priya et al. (2016) obtuvieron porcentajes de mortalidad de 53 y $58 \%$ utilizando extractos de frutos de Zanthoxyulum rhetsa Roxb.

El efecto insecticida producido por los EE de orégano y citronela sobre adultos de $R$. dominica, están asociados con la toxicidad 
ocasionada por los MS de ambos extractos, los cuales pudieron inducir inanición y/o asfixia del insecto. Adicionalmente, se deduce que la mayor cantidad de alcaloides, fenoles, flavonoides y saponinas del EE de citronela, pudo contribuir a que este extracto mostrara mayor efectividad en producir mortalidad. Al respecto, Ileke y Bulus (2012) mencionaron que los extractos pueden producir muerte por toxicidad de contacto o inducir asfixia por olores tóxicos y obstrucción de espiráculos, así como también pueden inhibir la locomoción, y en consecuencia reducir la capacidad para buscar alimento.

\section{Agradecimiento}

Al CDCHT - UCLA por el apoyo brindado a través del proyecto 1101-RAG-2017.

\section{Literatura Citada}

Abbott, W.

1925. A method of computing the effectiveness of an insecticide. Journal of Economy Entomology, 18: 265-267.

Acevedo, D.; Navarro, M.; Monrroy, L.

2013. Composición química del aceite esencial de hojas de orégano (Origanum vulgare). Información Tecnológica, 24 (4): 43-48.

Alonso, M.; Ávila, J.; Calcagno, M.

2009. Los cereales en el trópico suramericano: Técnicas modernas de conservación. CDCHT ULA - Fundación Empresas Polar. Caracas, Venezuela. p. 446.

Alvarado, S.; Ulacio, D., Sanabria, M., Pineda, J.

2011. Compatibilidad in vitro de extractos vegetales y Trichoderma harzianum y su efecto en el crecimiento de Sclerotium rolfsii Sacc., y Sclerotium cepivorum Berk. Boletín del Centro de Investigaciones Biológicas, 45 (3): 217-236.

Antolinez, J.; Colmenarez, N.; Usabillaga, A.; Darghan, E.; Linares, $\mathrm{S}$.

2008. Evaluación de variables agronómicas en el cultivo de limonaria (Cymbopogon citratus D.C) para la producción de aceite esencial. Interciencia, 33 (9): 693-699.

Avalos, A.; Pérez, E.

2009. Metabolismo secundario de plantas. Reduca (Biología). Serie Fisiología Vegetal, 2 (3): 119-145.

Avoseh, O.; Oyedeji, O.; Rungqu, P.; Nkeh-Chungag, B.; Oyedeji, A.

2015. Cymbopogon Species; Ethnopharmacology, Phytochemistry and the Pharmacological Importance. Molecules, 20: 7438-7453.

Bolívar, K.; Sanabria, M.; Rodríguez, D.; Camacaro, M.; Ulacio,

D.; Cumana, L.; Crescente, O.

2009. Potencial efecto fungicida de extractos vegetales en el desarrollo in vitro del hongo Colletotrichum gloeosporioides (Penz.) Penz. \& Sacc., y de la antracnosis en frutos de mango. Revista UDO Agrícola, 9 (1): 175-181.

Castañeda, M.; Muñoz, A.; Martínez, J.; Stanshenko, E. 2007. Estudio de la composición química y la actividad biológica de los aceites esenciales de diez plantas aromáticas colombianas. Scientia et Technica 13(33): 165-166.

Celis, A.; Mendoza, C.; Pachón, M.; Cardona, J.; Delgado,

W.; Cuca, L.E.

2008. Extractos vegetales utilizados como biocontroladores con énfasis en la familia Piperaceae. Una revisión. Agronomía Colombiana, 26 (1): 97-106.

Cuellar, A.; Márquez, I.; Hernández, J.; Alemán, A. 1999. Estudio fitoquímico de la especie Hibiscus elatus s.w. Revista Cubana de Farmacia, 33 (2): 127-31.
De Geyter, E.; Lambert, E.; Geelen, D.; Smagghe, G.

2007. Novel advances with plant saponins as natural insecticides to control pest insects. Pest Technology, 1 (2): 96-105.

Devine, G.J.; Dominique, E.; Ogusuku, E.; Furlong, M.J.

2008. Uso de insecticidas: contexto y consecuencias ecológicas. Revista Peruana de Medicina Experimental y Salud Pública, 25 (1): 74-100.

Fernández, O.; Sandoval, M.F.; Sanabria, M.E.; Vásquez, C.

2016. Efectividad in vitro del extracto etanólico de Cymbopogon citratus (D.L.) Stapf y Hexythiazox sobre Raoiella indica Hirst. IDESIA, 34 (2): 77-84.

Gandhi, N.; Pillai, S.

2011. Control of Rhyzopertha dominica (Coleoptera: Bostrichidae) by pulverized leaves of Punica granatum (Lythraceae) and Murraya koenigii (Rutaceae). International Journal of Agriculture and Biology, 13 (4): 535-540.

Ileke, K.; Bulus, D.

2012. Response of lesser grain borer, Rhyzopertha dominica (Fabr.) (Coleoptera: Bostrichidae) to powders and extracts of Azadirachta indica and Piper guineense seeds. Jordan Journal of Biological Sciences, 5 (4): 315-320.

Khaliq, A.; Javed, M.; Hannan, M.; Yasir, M.; Sangheer, M.; Hasan, M.

2014. Biocidal and repellent effect of Saccharopolyspora spinosa and Nerium oleander extract against stored product pests. Basic Research Journal of Agricultural Science and Review, 3 (6): 49-54.

Lima, A.; Broglio, S.M.F.; Araujo, A.M.N.; Lopes, D.O.P.; Dias-Pini, N.S.

2013. Efeito de pós vegetais sobre Sitophilus zeamais (Mots., 1855) (Coleoptera: Curculionidae). Arquivos do Instituto Biológico, 80 (1): 91-97.

Marcano, D.; Hasegawa, M.

2002. Fitoquimica orgánica. Universidad Central de Venezuela (UCV). Consejo de Desarrollo Científico Humanístico y Tecnológico (CDCHT). Editorial Torino. 1era edición. Caracas- Venezuela. 588 p.

Olivero, J.; Caballero, K.; Jaramillo, B.; Stashenko, E.

2009. Actividad repelente de los aceites esenciales de Lippia origanoides, Citrus sinensis y Cymbopogon nardus, cultivadas en Colombia frente a Tribolium castaneum Herbst. Revista de la Universidad Industrial de Santander. Salud, 41 (3): 244-250.

Pino, O.; Sánchez, Y.; Rojas, M.

2013. Plant secondary metabolites as alternatives in pest management. II: An overview of their potential in Cuba. Revista de Protección Vegetal, 28 (2): 95-108. 
Priya, A.; Trupti, S.; Mira, R.; Aparna S.

2016. Control of Rhyzopertha dominica by various solvents extracts of fruits of Zanthoxyulum rhetsa Roxb dc (Rutaceae). International Journal of Recent Scientific Research, 7 (6): 12061-12070.

Rajendran, S.; Gunasekaran, N.

2002. The response of phosphine-resistant lesser grain borer Rhyzopertha dominica and rice weevil Sitophilus oryzae in mixed-age cultures to varying concentrations of phosphine. Pest Management Science, 58 (3): 277-281.

Reyes-Guzmán, R.; Borboa-Flores, J.; Cinco-Moroyoqui, F.J.; Rosas-Burgos, E.C.; Osuna-Amarillas, P.S.; Wong-Corral, F.J.; Ortega-Nieblas, M.M.; León-Lara, J.D.

2012. Actividad insecticida de aceites esenciales de dos especies de Eucalyptus sobre Rhyzopertha dominica y su efecto en enzimas digestivas de progenies. Revista Chapingo Serie Ciencias Forestales y del Ambiente, 18 (3): 385-394.
Sepúlveda, G.; Porta, H.; Rocha, M.

2003. La participación de los metabolitos secundarios en la defensa de las plantas. Revista Mexicana de Fitopatología, 21 (3): 355-363.

Schlipalius, D.; Cheng, Q.; Reilly, P.; Collins, P.; Ebert, P. 2002. Genetic linkage analysis of the lesser grain borer Rhyzopertha dominica identifies two loci that confer high-level resistance to the fumigant phosphine. Genetics, 161 (2): 773-782.

Sivira, A.; Sanabria, M.E.; Valera, N.; Vásquez, C.

2011. Toxicity of ethanolic extracts from Lippia origanoides and Gliricida sepium to Tetranychus cinnabarinus (Boisduval) (Acari: Tetranychidae). Neotropical Entomology, 40 (3): 375 -379.

Vásquez, C.; Aponte, O.; Morales, J.; Sanabria, M.; García, G. 2008. Biological studies of Oligonychus punicae (Acari: Tetranychidae) on grapevine cultivars. Experimental and Applied Acarology, 45 (1-2): 59-69. 